\title{
Aloperine executes antitumor effects through the induction of apoptosis and cell cycle arrest in prostate cancer in vitro and in vivo
}

This article was published in the following Dove Press journal:

OncoTargets and Therapy

\author{
Zhixin Ling ${ }^{1,2, *}$ \\ Han Guan ${ }^{3, *}$ \\ Zonghao You ${ }^{1,2, *}$ \\ Can Wang ${ }^{1,2}$ \\ Ling $\mathrm{Hu}^{4}$ \\ Lei Zhang ${ }^{1,2}$ \\ Yiduo Wang ${ }^{1,2}$ \\ Shuqiu Chen ${ }^{1,2}$ \\ Bin $X u^{1,2}$ \\ Ming Chen ${ }^{1,2}$ \\ 'Department of Urology, Affiliated \\ Zhongda Hospital of Southeast \\ University, Nanjing, People's Republic \\ of China; ${ }^{2}$ Surgical Research Center, \\ Institute of Urology, Medical School of \\ Southeast University, Nanjing, People's \\ Republic of China; ${ }^{3}$ Department \\ of Urology, The First Affiliated \\ Hospital of Bengbu Medical College, \\ Bengbu, People's Republic of China; \\ ${ }^{4}$ Department of Nephrology, People's \\ Hospital of Wuxi City, Wuxi, People's \\ Republic of China \\ *These authors contributed equally \\ to this work
}

\begin{abstract}
Background: Prostate cancer (PCa) is one of the most common malignant diseases among male patients. Although androgen deprivation therapy remains the main treatment for $\mathrm{PCa}$, most patients would inevitably progress to castration-resistant $\mathrm{PCa}$, which is the main cause of cancer-related deaths. Thus, novel antitumor agents are urgently needed. Recent studies demonstrated that aloperine (ALO) as a natural alkaloid showed antitumor effects in other cancer types. However, the biological function and underlying mechanisms of ALO in PCa have not been investigated.
\end{abstract}

Methods: PCa cell lines including LNCaP, PC3 and DU145 were cultured and treated with ALO. Cell Counting Kit-8 assay, colony formation assay, apoptosis assay and cell cycle assay were conducted to assess the biological role of ALO. In addition, a PCa subcutaneous xenograft mouse model was established to evaluate the role of ALO in terms of proliferation and apoptosis in vivo. We further measured the protein expression levels of p-Akt/Akt, p-ERK/ ERK, c-Myc, cleaved caspase 3, p21, p53, Bcl-2 and Bax using the Western blot $48 \mathrm{~h}$ after ALO treatment of PCa cells.

Results: ALO effectively inhibited the cell viability of PCa by inducing cell cycle arrest via the activation of the p53/p21 pathway and triggering apoptosis in vitro and in vivo. ALO also inhibited phosphorylation of Akt and ERK protein kinases and activated cleaved caspase 3 while exerting antiproliferation function through inducing apoptosis and cell cycle arrest in PCa cells

Conclusion: Based on our findings, we conclude that ALO could suppress the tumor growth and promote cell apoptosis and cell cycle arrest in PCa cells, which indicated that ALO could act as a novel therapeutic agent in treatment of human PCa.

Keywords: aloperine, prostate cancer, proliferation, apoptosis, cell cycle

\section{Introduction}

Prostate cancer $(\mathrm{PCa})$ is expected as the most common diagnosed cancer as well as one of the most common causes of cancer death among men in America. ${ }^{1}$ The incidence and mortality rates of $\mathrm{PCa}$ in China have been continuously increasing in recent years. ${ }^{2}$ Although androgen deprivation remains to be the standard therapy for localized $\mathrm{PCa}$, patients still progress to castration-resistant PCa despite low levels of circulating androgens which is the main cause of cancer-related deaths. The development of second-generation antiandrogen drugs including enzalutamide and abiraterone seems to be a promising therapeutic approach for advanced $\mathrm{PCa}$; however, studies have reported that $A R$ mutations or aberrantly increased androgen receptor expression levels could confer resistance to these drugs. ${ }^{3,4}$ Thus, comprehensive treatment strategies including
Correspondence: Bin Xu; Ming Chen Department of Urology, Affiliated Zhongda Hospital of Southeast University, No 87, Dingjiaqiao, Gulou, Nanjing 210009 , Jiangsu, People's

Republic of China

Tel +86 I80 I294 9196;

+86 I39 I3009977

Email njxb1982@126.com;

mingchenseu@।26.com 
novel therapeutic drugs are needed to improve the overall survival rate and life quality of PCa patients.

Aloperine (ALO) was first reported as an alkaloid isolated from Sophora alopecuroides L. which showed strong anti-inflammatory and anti-allergic functions. ${ }^{5,6}$ ALO exhibits protective effects on neonatal rat primary cultured hippocampal neurons and ischemia reperfusioninduced injury induced by oxygen-glucose deprivation and reperfusion. ${ }^{7,8}$ Interestingly, several studies reported that ALO also exerts antitumor effects on multiple malignant neoplasms including colon cancer, myeloma, lung carcinoma and osteosarcoma. ${ }^{9-12}$ Therefore, ALO has different therapeutic effects on benign inflammatory diseases and malignant cancers. However, there are no studies till date illustrating the biological effects of ALO on PCa cells and its underlying mechanisms in these activities.

This present study aims to systematically investigate the antitumor effects of ALO against PCa cells in vitro and in vivo, and elucidate its underlying molecular mechanisms responsible for apoptosis and cell cycle arrest in ALOinduced cytotoxicity.

\section{Materials and methods}

\section{Cell lines and chemicals}

The androgen-dependent human PCa cell line LNCaP and androgen independent $\mathrm{PCa}$ cell lines PC 3 and DU145 were purchased from ATCC (Manassas, VA, USA). All these cell lines were cultured in the RPMI-1640 medium (Thermo Fisher Scientific, Waltham, MA, USA) supplemented with $10 \%$ fetal bovine serum (Thermo Fisher Scientific), $100 \mathrm{U} / \mathrm{mL}$ penicillin and $100 \mu \mathrm{g} / \mathrm{mL}$ streptomycin in humidified air at $37^{\circ} \mathrm{C}$ with $5 \% \mathrm{CO}_{2}$.

ALO with a HPLC-grade purity of $98 \%$ was purchased from Selleck (Houston, TX, USA) and it was dissolved in DMSO and stored at $-20^{\circ} \mathrm{C}$ until used. All these cells were treated with the ALO premixed cell culture medium in which the final concentration of DMSO did not exceed $0.1 \%$. The molecular structure of ALO is shown in Figure 1A.

\section{Cell Counting Kit-8 assay}

LNCaP, PC3 and DU145 cells at 3,000 cells/well in $100 \mu \mathrm{L}$ of the complete medium were seeded in 96-well plates. After culturing for $24 \mathrm{~h}$, the medium was replaced with a fresh complete medium containing different concentrations of $\operatorname{ALO}(0,100$ and $200 \mu \mathrm{M})$. Each concentration was repeated with five wells, and PCa cells were exposed to ALO for 24, 48, 72 and $96 \mathrm{~h}$. Cell proliferation was measured every $24 \mathrm{~h}$ using the Cell Counting Kit-8 (CCK-8; KeyGene Biotech, Nanjing, People's Republic of China) according to the manufacturer's instruction. The absorbance at $450 \mathrm{~nm}$ was measured using a microplate reader (Thermo Fisher Scientific, Waltham, MA, USA). Three independent experiments were each carried out with a 96-well plate.

\section{Cell colony formation assay}

All the cell lines were cultured in a six-well plate with three different concentrations of $\operatorname{ALO}(0,100,200 \mu \mathrm{M})$ for 10 days as we have previously described..$^{13}$ Then cells were fixed with methanol and stained with $0.5 \%$ crystal violet. The number of colonies was counted when they contained more than 50 cells.

\section{Morphology observation}

PC3, DU145 and LNCaP cells were grown in six-well plates and cultured with different concentrations of $\operatorname{ALO}(0,100$, $200 \mu \mathrm{M}$ ) for $12 \mathrm{~h}$. Subsequently, the images of morphological changes of these cells were captured using a microscope (Nikon, Tokyo, Japan).

\section{Cell cycle and apoptosis assay}

Cells were cultured with three gradient densities of ALO in a six-well plate for $48 \mathrm{~h}$. For the cell cycle assay, cells were collected and fixed with $75 \%$ cold ethanol at $-20^{\circ} \mathrm{C}$ overnight. Subsequently, cells were stained with $50 \mu \mathrm{g} / \mathrm{mL}$ of propidium iodide (PI) and $1 \mathrm{mg} / \mathrm{mL}$ RNase for $30 \mathrm{~min}$ at $4^{\circ} \mathrm{C}$. Cell cycle distributions were analyzed using a FACS flow cytometer (BD Biosciences, San Jose, CA, USA). As for the apoptosis assay, cells were stained with Annexin V-FITC/PI which was contained in an apoptosis kit (KeyGene Biotech). Flow cytometry was then performed and results were analyzed.

\section{Xenograft model study}

The study was approved by the Animal Care Committee of the Medical School of Southeast University and mice were treated according to the National Institutes of Health Guide for the Care and Use of Laboratory Animals. A total of 10 male BALB/C nude mice (6 weeks old) were purchased from the Model Animal Research Center of Nanjing University. All mice were subcutaneously injected with PC3 cells $\left(5 \times 10^{6}\right.$ cells per mice) and randomized into ALO (30 mg/kg, p.o., daily) and control (PBS, p.o., daily) groups (five mice per group). The tumor volumes were measured every 4 days (length $\times$ width $^{2} / 2$ ). At the end of experiments, all mice were sacrificed and tumors were dissected and weighed. Then, tissues were fixed with formalin and embedded with paraffin. 
A

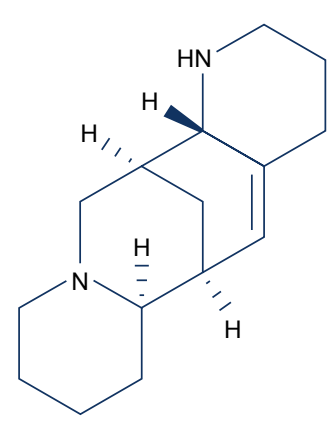

B

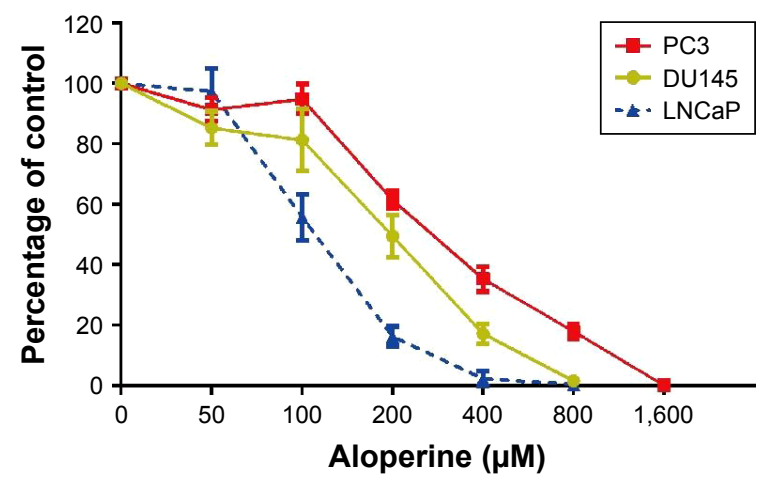

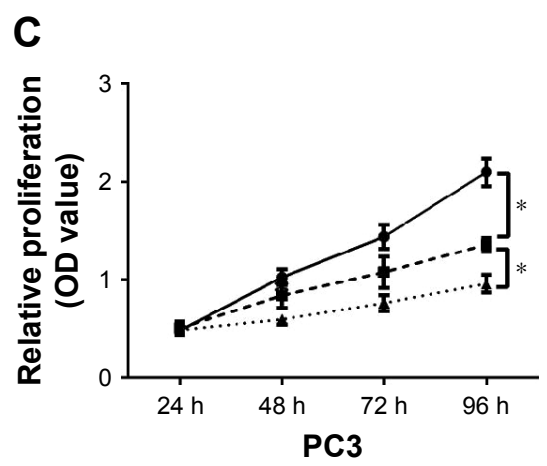

D
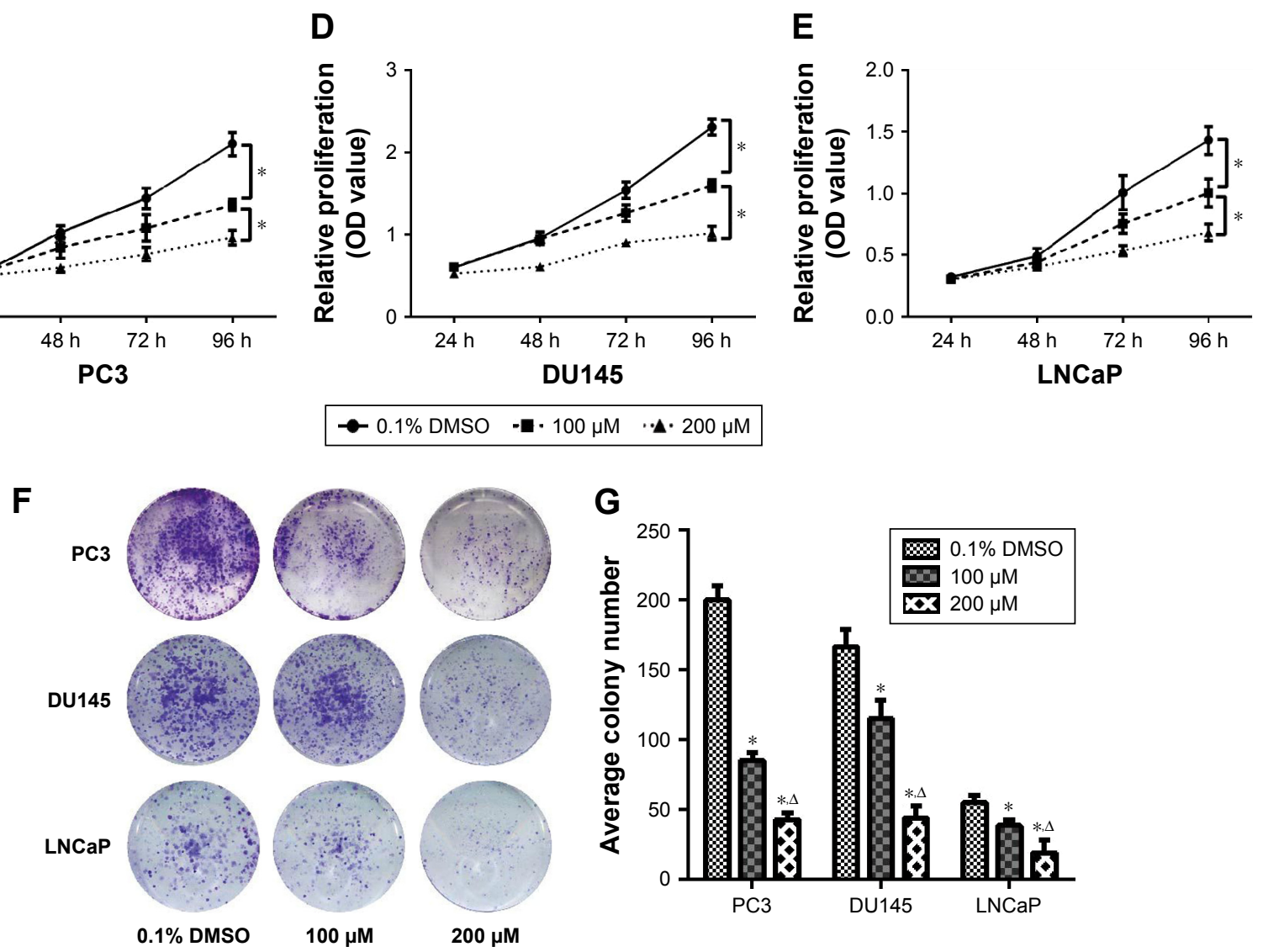

G
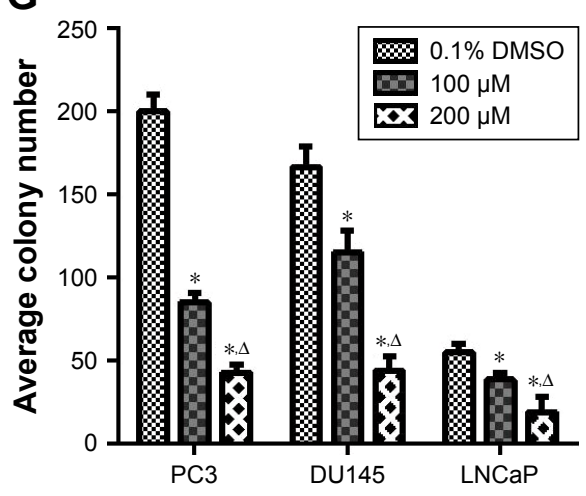

Figure I Aloperine inhibited the proliferation of prostate cancer cells in vitro. (A) The molecular structure of aloperine provided by the manufacturer. (B) Prostate cancer cell lines were treated with the indicated dose of aloperine or vehicle for $72 \mathrm{~h}$, and a CCK-8 assay was performed to calculate IC50 values of aloperine for different cell lines. (C) CCK-8 assay for PC3 cells exposed to aloperine ( 100 and $200 \mu \mathrm{M})$ for $24,48,72$ and $96 \mathrm{~h}(* \mathrm{P}<0.05, \mathrm{n}=5)$. (D) CCK-8 assay for DUI45 cells exposed to aloperine $(\mathrm{I} 00$ and $200 \mu \mathrm{M})$ for $24,48,72$ and $96 \mathrm{~h}(* \mathrm{P}<0.05, \mathrm{n}=5)$. (E) CCK-8 assay for $\mathrm{LNCaP}$ cells exposed to aloperine $(\mathrm{I} 00$ and $200 \mu \mathrm{M})$ for $24,48,72$ and $96 \mathrm{~h}(* \mathrm{P}<0.05, \mathrm{n}=5)$. (F) Colony formation assay for PC3, DUI45 and LNCaP cells exposed to aloperine ( 100 and $200 \mu \mathrm{M})$ or vehicle for 10 days. (G) Quantitative analysis of clone formation for PC3, DUI45 and LNCaP cells treated with aloperine and untreated cells $\left(* P<0.05\right.$ vs vehicle, ${ }^{\Delta P}<0.05$ vs $\left.100 \mu M, n=5\right)$.

Abbreviation: CCK-8, Cell Counting Kit-8.

\section{TUNEL assay and immunohistochemical staining}

The tumor tissues (both treatment and control group) were stained with antibody against Ki-67 (1:100; Boster Biological Technology, Ltd., Wuhan, People's Republic of China) to evaluate the proliferation rate of PCa cells in vivo. Moreover, tumor tissue apoptotic cells were assessed by a terminal deoxynucleotidyl transferase-mediated dUTP nick-end labeling
(TUNEL) assay using an In Situ Cell Death Detection Kit (Roche, Mannheim, Germany). All images were captured using a microscope (Nikon). The apoptotic score was calculated by counting the number of positive nuclei in 30 random fields.

\section{Western blot analysis}

Total protein was obtained from PCa cells lysed with the RIPA buffer (50 mM Tris-HCl, pH 8.0; 150 mM NaCl, 
5 mM EDTA, 0.1\% SDS, 1\% NP-40) supplemented with 1\% proteinase inhibitors (Sigma-Aldrich, St Louis, MO, USA) and $1 \mathrm{mM}$ PMSF (Beyotime, Hangzhou, People's Republic of China). Then samples were subjected to sodium dodecyl sulfate-polyacrylamide gel electrophoresis and transferred into a polyvinlidene fluoride membrane (Merck Millipore, Billerica, MA, USA). After blocking with 5\% skim milk for $60 \mathrm{~min}$, blots were incubated with specific primary antibodies: anti-p-AKT, anti-AKT (Thermo Fisher Scientific), antip-ERK, anti-ERK, anti-c-Myc (Cell Signaling Technology, Danvers, MA, USA), anti-cleaved caspase 3, anti-Bcl-2, anti-Bax (Abcam, Cambridge, UK), anti-p21, anti-p53 and anti-GAPDH (Proteintech, Rosemont, IL, USA). After washing the membranes three times with the TBST buffer (a mixture of TBS-1\% and Tween 20), blots were incubated with corresponding secondary antibodies for $1 \mathrm{~h}$ and detected with enhanced chemiluminescence (Beyotime).

\section{Statistic analysis}

All data in this study were presented as means \pm standard deviations and experiments mentioned above were repeated at least three times. IC50 values for proliferation were calculated as the indicated concentration of ALO that reduced half viable cells compared with the control group using the CCK-8 assay. The significance of difference among the means was calculated using Student's $t$-tests for two groups using SPSS 19.0 software. A two-sided $P$-value $<0.05$ was considered to be statistically significant.

\section{Results}

\section{ALO inhibited the proliferation of $\mathrm{PCa}$ cells in vitro}

To assess the biological effects of ALO on PCa cells, we first evaluated the cell viability of PCa cells after treatment with different concentrations of ALO for $72 \mathrm{~h}$. Results indicated that ALO inhibited the cell proliferation of both androgen-dependent and independent PCa cell lines in a dose-dependent manner (Figure 1B). The IC50 value of ALO was $100-300 \mu \mathrm{M}$ for all PCa cell lines. In addition, ALO suppressed cell viability of PCa cells in a time-dependent manner when we treated PC3, DU145 and LNCaP with ALO ( 100 or $200 \mu \mathrm{M}$ ) for $24,48,72$ and $96 \mathrm{~h}$. The inhibition efficiency of ALO was significantly different from that of vehicle (0.1\% DMSO)-treated control group after $96 \mathrm{~h}$ (Figure $1 \mathrm{C}-\mathrm{E}$, $P<0.05)$. Moreover, the proliferation rate of $\mathrm{PCa}$ cells was significantly different between two concentrations of ALO (100 and $200 \mu \mathrm{M})$-treated groups $(P<0.05)$. Similarly,
ALO treatment also significantly suppressed the cell viability of PCa based on the colony formation assay (Figure 1F). Furthermore, the reduction of cell proliferation was more obvious in $200 \mu \mathrm{M}$ of the ALO-treated group than that of $100 \mu \mathrm{M}$ of the ALO-treated group (Figure $1 \mathrm{G}, P<0.05$ ).

\section{ALO-induced apoptosis and cell cycle arrest of $\mathrm{PCa}$ cells in vitro}

PC3, DU145 and LNCaP cells were observed using a microscope at $200 \times$ magnification after they were exposed to $\operatorname{ALO}(0,100$ and $200 \mu \mathrm{M})$ for $12 \mathrm{~h}$. The results demonstrated that the number of all the PCa cell lines was gradually decreased. Meanwhile, the morphological changes of cells including cell shrinkage and cell surface irregularity indicated ALO may have an apoptotic effect on PCa cells (Figure 2A). Then we further performed the Annexin-V/FITC apoptosis assay to investigate whether ALO could induce PCa apoptosis. After being exposed to various concentrations of ALO (100 and $200 \mu \mathrm{M}$ ) for $48 \mathrm{~h}$, the percentage of Annexin-V/ FITC-positive cells was found to be significantly increased compared with that of respective control group (Figure 2B). Moreover, the percentage of apoptotic cells increased along with gradient concentration in PC3, DU145 and LNCaP cells (Figure 2C). The cell cycle assay demonstrated that ALO evidently induced G1 phase arrest accompanied with $\mathrm{S}$ phase proportion reduction in PCa cells when compared with vehicle-treated cells. Meanwhile, no significant difference was observed in the fraction of the G2 phase in $\mathrm{PCa}$ cells between ALO-treated and vehicle-treated groups except for a small reduction in the G2 phase in PC3 cells exposed to $200 \mu \mathrm{M}$ of ALO (Figure 2D-G).

\section{ALO suppressed the formation of prostate xenograft tumors in vivo}

To further investigate whether ALO possesses the tumor suppressive ability in vivo, we performed the BALB/c nude mice xenograft assay by subcutaneous inoculation of PC3 cells following with a dose of $30 \mathrm{mg} / \mathrm{kg}$ of ALO or PBS per o.s. daily. Results revealed that ALO remarkedly suppressed the PC3 tumor growth as manifested reduced tumor volume and tumor weight at the end of the monitoring time (Figure 3A-D). The difference in the tumor volume between ALO-treated and PBS-treated groups became significant from 12 days after PC3 cell injection. No significant difference was observed in the weights of mice between treatment and control groups (Figure 3E). Immunohistochemical (IHC) staining and TUNEL assays showed that reduced Ki-67 expression and increased apoptotic cells were observed in 

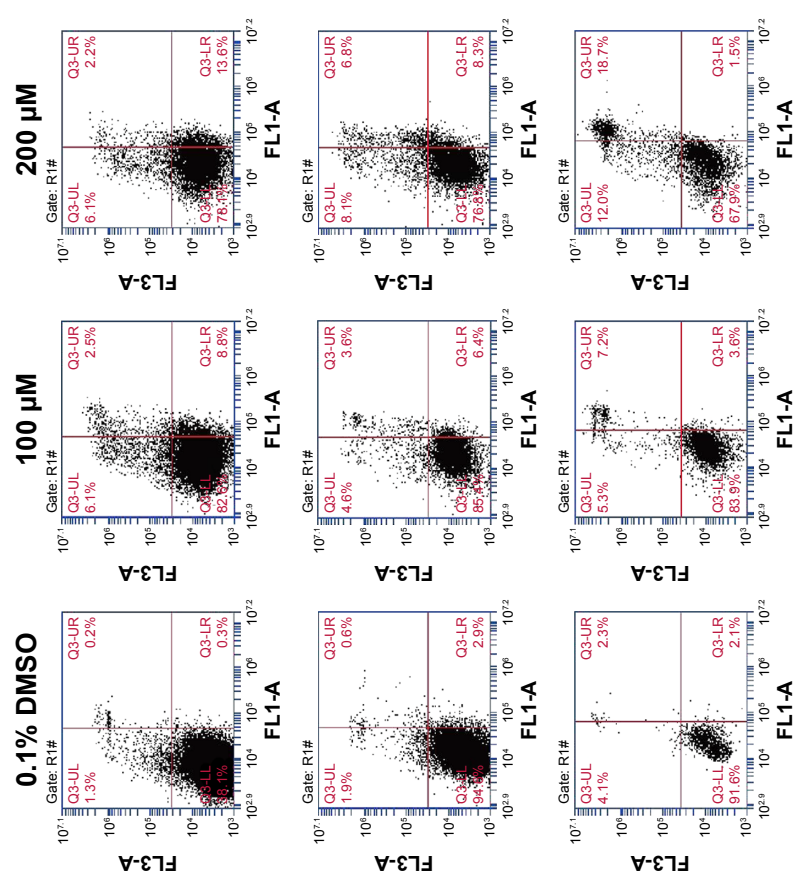

$\infty \quad \varepsilon 0 d$

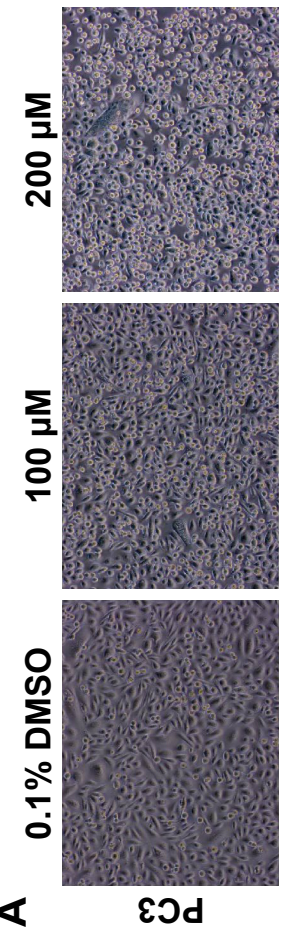

Strna
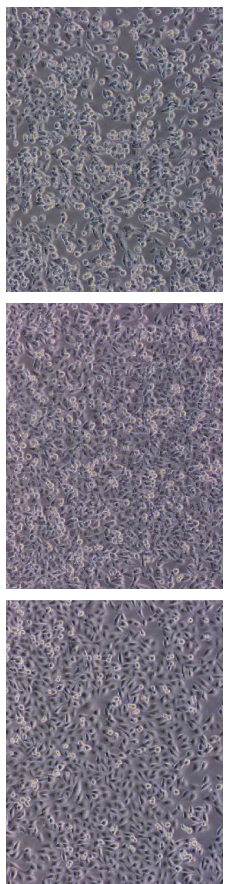

SャIna
deכNר
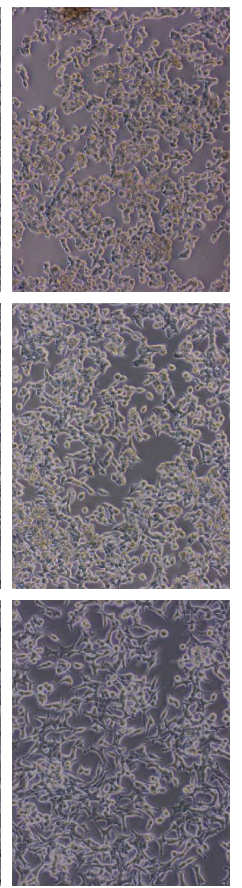

deכNר
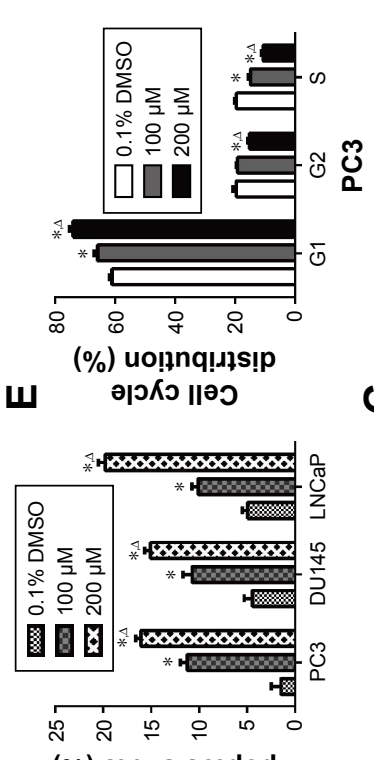

(\%) әॄеג s!̣soldod $\forall$

0
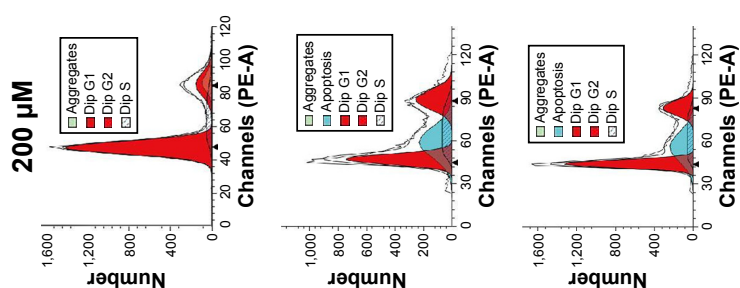

(\%) uo!̣nq!n?!

0 ә әКэ ॥ә弓

뜽

응

乙要要

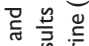

守感

辡远

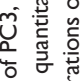

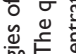

이의

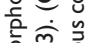

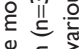

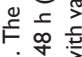

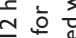

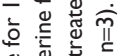

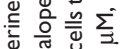

응 응응

둥

造

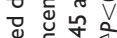

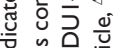

흥

政

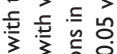

造

(\%) uounnqusp

әગरิ ॥әว

ᄂ

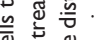

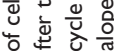

递

o응

은 월 品

ह व

品骂它

焉

区

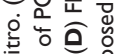

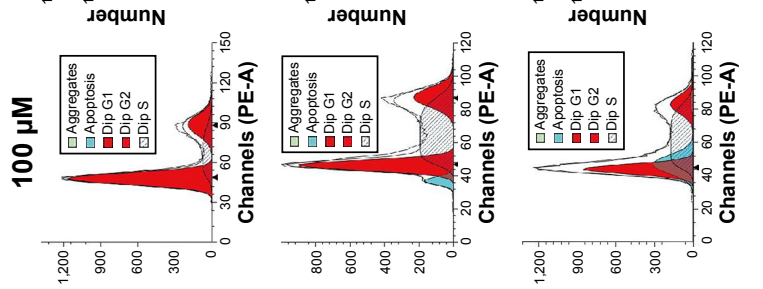

岛

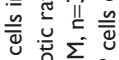

항응

(ึ)

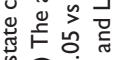

ํㅜㄴ

o
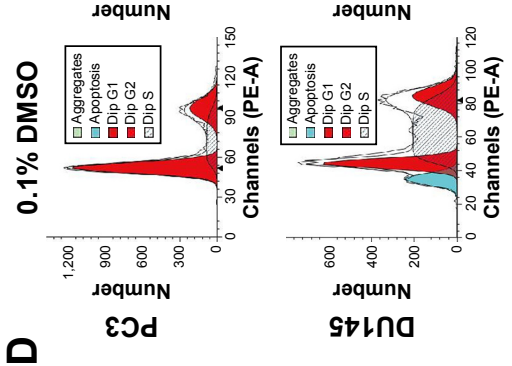

苍至它

के 을

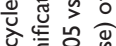

要

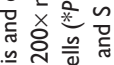

号过

응 ڤٓ

त्

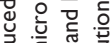

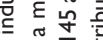

我

ڤั웡

造产员

웜 은

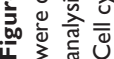


A
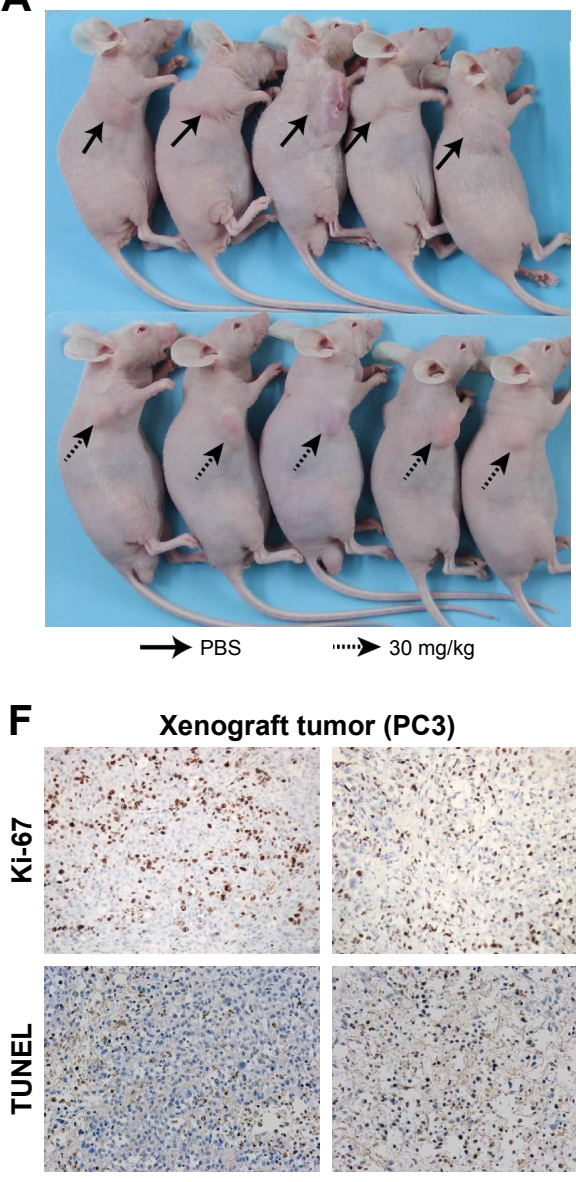

PBS
B

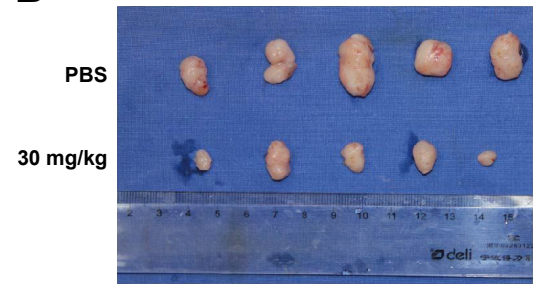

D

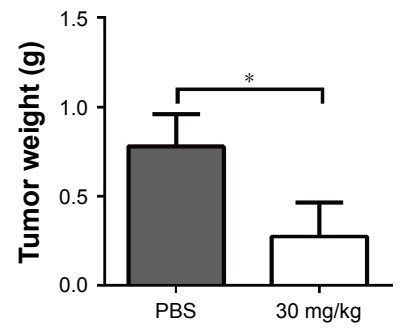

G

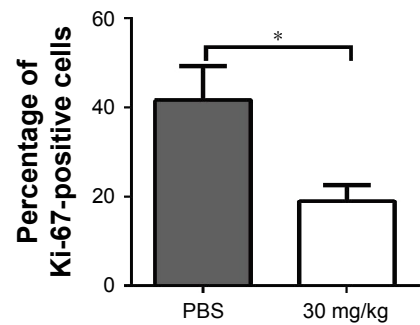

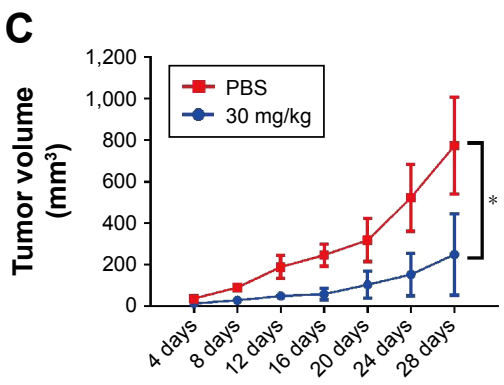

E
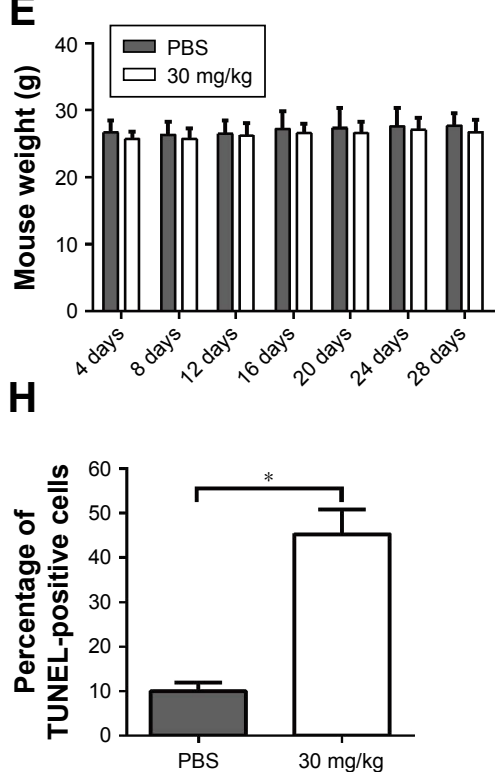

Figure 3 Aloperine suppressed PC3 xenograft tumor growth in vivo. (A and B) PC3 cells were injected into the right flank of 6-week-old male, nude mice. Subcutaneous tumors were formed in nude mice after treatment with either PBS or aloperine $(30 \mathrm{mg} / \mathrm{kg})$ for 28 days. (C) Tumor volume of aloperine-treated PC 3 cells at the indicated time $(* P<0.05$ vs vehicle, $n=5)$. (D) Histograms describing the mean tumor weights of each group $(* P<0.05$ vs vehicle, $n=5)$. (E) Mice weight at the indicated time. (F) Immunohistochemical staining of Ki-67 and TUNEL assay in the endpoint tumors. (G and $\mathbf{H}$ ) Quantitative analysis of Ki-67-positive and TUNEL-positive cells in tumor tissues of different groups ( $* P<0.05$ vs vehicle, $n=5)$.

Abbreviation: TUNEL, terminal deoxynucleotidyl transferase-mediated dUTP nick-end labeling.

the tumor tissues treated with ALO compared with PBS (Figure $3 \mathrm{~F}-\mathrm{H}, P<0.05$ ).

\section{ALO suppressed AKT and ERK phosphorylation, activated caspase 3 and induced activation of the $\mathrm{p} 53 / \mathrm{p} 2 \mathrm{I}$ pathway} Previous studies have reported that AKT and ERK signal pathways are implicated in apoptosis. ${ }^{14,15}$ Moreover, the p53/p21 pathway is an important negative regulator of cell cycle progression. ${ }^{16,17}$ And p53-dependent transcriptional repression of c-Myc is required for G1 phase arrest. ${ }^{18}$ Therefore, we performed Western blot assay to assess the expression level of these apoptosis- and cell cycle-related proteins (p-Akt/ Akt, p-ERK/ERK, cleaved caspase 3, p53, p21, c-Myc, Bcl-2, $\mathrm{Bax}$ ) to further illustrate the molecular mechanisms by which ALO suppressed the PCa cell growth (Figure 4A). Results showed that ALO significantly suppressed the phosphorylation of Akt and ERK which led to the reduction of expression of p-Akt and p-ERK when compared with the vehicle-treated group. However, no significant difference was observed in the total expression of Akt and ERK between ALO-treated and vehicle-treated PCa cells. Moreover, ALO activated the caspase 3 which dramatically elevated the expression of cleaved caspase 3 in PCa cells after exposure to ALO. ALO also suppressed the expression of anti-apoptotic protein $\mathrm{Bcl}-2$ and upregulated that of pro-apoptotic protein $\mathrm{Bax}$ in $\mathrm{PCa}$ cells. Another important mechanism is that the activation of p53/p21 pathway by ALO induced G1 phase cell cycle arrest in all PCa cell lines. The expression levels of p53 and p21 both elevated when cells were treated with ALO while c-Myc as a target gene of 553 was transcriptionally repressed (Figure 4B-D). 


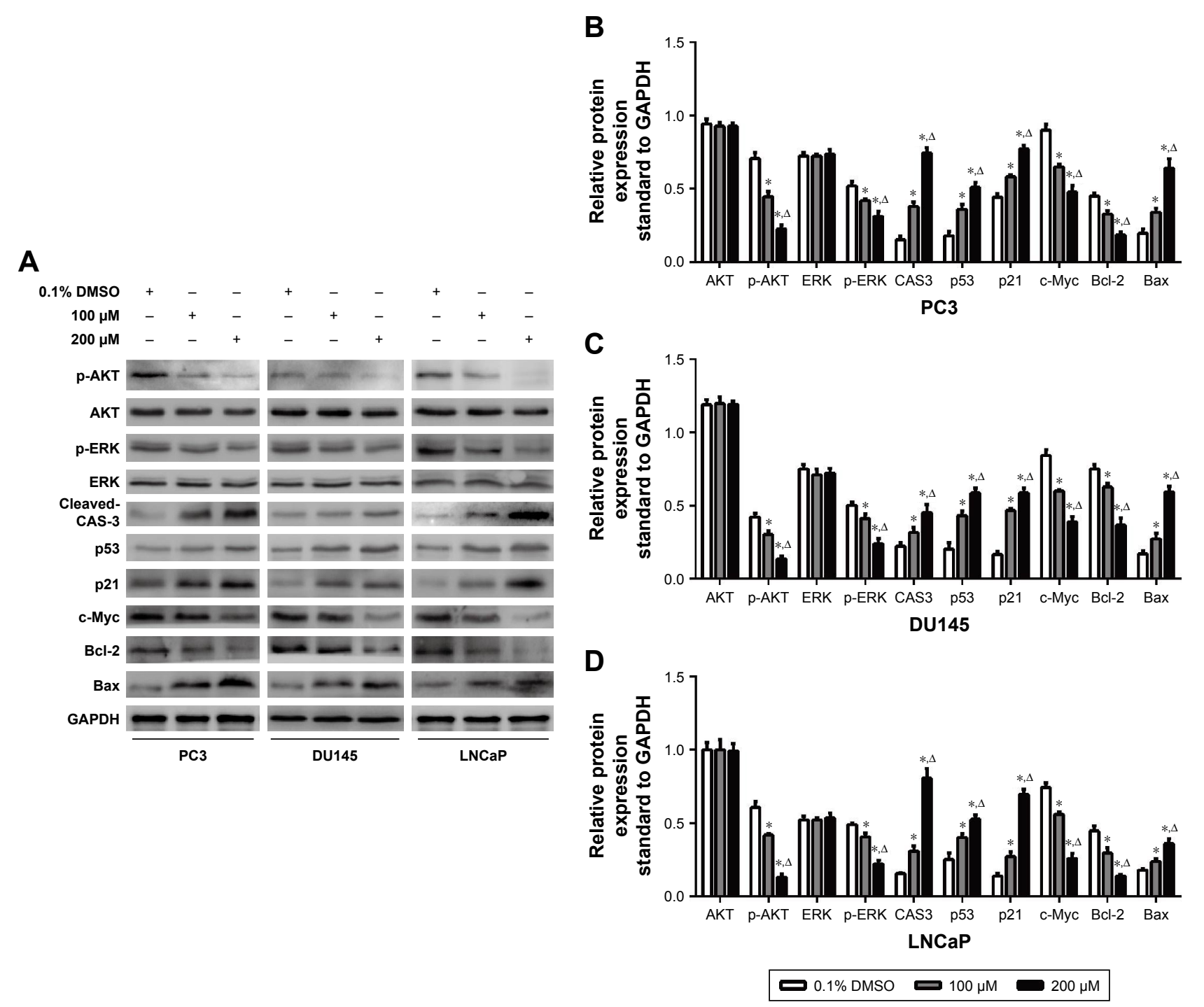

Figure 4 Western blot analysis. (A) Lysates from control and aloperine-treated PC3, DUI 45 and LNCaP cells were subjected to Western blot analysis using anti-p-AKT, AKT, p-ERK, ERK, c-Myc, cleaved caspase 3 (CAS3), Bcl-2, Bax and GAPDH. (B-D) Quantitative analysis of protein expression levels in prostate cancer cells treated with vehicle or aloperine $\left(* P<0.05\right.$ vs vehicle, ${ }^{\Delta P}<0.05$ vs $\left.100 \mu \mathrm{M}, \mathrm{n}=3\right)$.

\section{Discussion}

Although the prognosis of PCa patients has been improved significantly due to the development of various therapeutic approaches, the effective managements against castrationresistant $\mathrm{PCa}$ to date remain limited. Therefore, there is an urgent need to explore novel chemical agents that execute strong antitumor effects against PCa. Chinese traditional medicine has been widely used to treat various diseases for thousands of years. ALO, a natural alkaloid constituent isolated from the herb $S$. alopecuroides, has been reported to exhibit antiproliferative activity against colon cancer SW480 and HCT116 cell lines, myeloma cell lines and human osteosarcoma cell lines (MG-63 and U2OS). ${ }^{9,10,12}$ In this study, we first investigated the inhibitory effects of ALO against both androgen-dependent and independent PCa cell lines ( $\mathrm{LNCaP}$, PC3 and DU145) in vitro and in vivo. Our data showed that ALO inhibited the growth of PCa cell lines at low IC50 values in dose- and time-dependent manners. Moreover, ALO could effectively inhibit the clonogenic survival of PCa cell lines by inducing apoptosis and cell cycle arrest. The in vivo study demonstrated that ALO dramatically suppressed the PCa tumor growth which revealed that ALO may be a novel therapeutic agent for PCa. Numerous studies have demonstrated that a lot of chemical agents showed antitumor activities via triggering apoptosis and cell cycle arrest, ${ }^{19-21}$ apoptosis and cell cycle arrest as important cytotoxic effects for most anticancer drugs. However, the underlying mechanisms of ALO-induced antitumor activities in PCa remain unclear. 
A previous study has shown that ALO may induce G2/M phase cell cycle arrest in HCT116 human colon cancer. ${ }^{9}$ In the present study, we also investigated the cycle distribution by using flow cytometry. Interestingly, the data showed that the proportion of G1 phase cells increased in a dose-dependent manner, indicating ALO inhibited PCa cell growth through G1 phase arrest. These results indicated that ALO had a master regulatory function in cell cycles. Progression through the cell cycle is controlled by the cyclin-dependent kinase (CDK) family of serine/threonine kinases and their regulatory partners, the cyclins. ${ }^{22} \mathrm{G} 1$ phase is driven by cyclin D-CDK4, cyclin D-CDK6 and cyclin E-CDK2 through the restriction point, which commits the cell to complete the cycle. ${ }^{23} \mathrm{P} 21$ was characterized as a crucial CDK inhibitor which could bind CDK4, CDK6 and cyclin D-CDK4 (or CDK6) or cyclin E-CDK2 complex. ${ }^{24,25}$ P53 has also been reported to control both G1 and G2/M cell cycle checkpoints and mediate growth arrest in human cells. ${ }^{26,27}$ P21 however is largely controlled by tumor suppressor p53; thus, the p53/p21 pathway played a vital role in the regulation of cell cycle. Another study has also proven that $\mathrm{p} 53$-mediated c-Myc repression is required for the p53-dependent G1 phase cell cycle arrest. ${ }^{18}$ The present study has shown that ALO upregulated the expression levels of p53 and p21 and suppressed the expression of c-Myc in three PCa cell lines, which may lead to the cell cycle arrest. Moreover, Ki-67 is a very popular proliferation marker and is routinely used in pathology labs due to its diagnostic and prognostic power in cancer. It is preferentially expressed during the late G1, S, G2 and M phases of the cell cycle, whereas resting, noncycling cells (G0 phase) lack Ki-67 expression. ${ }^{28}$ We performed the IHC staining of Ki-67 of xenograft tumor tissues treated with ALO or PBS. The expression of Ki-67 significantly decreased in the PC3 xenograft tumor treated with ALO which proved that ALO also inhibited PCa cell proliferation via inducing cell cycle arrest in vivo.

In addition to cell cycle arrest, ALO also exhibited pro-apoptotic properties both in vitro and in vivo. After incubation with ALO, we observed the cell morphological changes in PC3, DU45 and LNCaP cells which indicated that ALO induced apoptosis in PCa cells. Then these results were confirmed by the flow cytometry assay showing that the proportion of Annexin-V/PI-positive cells increased along with the higher concentrations of ALO. The TUNEL assay also indicated that the apoptosis rate was increased in xenograft tumor tissues when mice were treated with ALO. The PI3K/Akt and Ras/Raf/MEK/ERK pathways are the central signal transduction mechanisms for controlling cell proliferation, survival, metabolism and apoptosis in response to extracellular stimuli. $\mathrm{Hu}$ et $\mathrm{al}^{29}$ found that the inhibition of both AKT and ERK1/2 pathways greatly sensitized LNCaP, PC3 and DU145 cells to apoptosis induced by methylseleninic acid. Our data revealed that ALO could significantly suppress the phosphorylation of Akt and ERK in a dose-dependent manner while no significant difference was observed in the total expression levels of Akt and ERK between different groups. Moreover, ALO treatment also increased the expression of cleaved caspase 3 in PCa cells. It is known that a shift in the $\mathrm{Bax} / \mathrm{Bcl}-2$ ratio could activate caspase 3 and induce apoptosis. ${ }^{30}$ Then we further assessed the expression levels of pro-apoptotic protein Bax and antiapoptotic protein Bcl-2. The Western blot assay showed that ALO treatment upregulated Bax and downregulated Bcl-2 in PCa cells. All these results explained why ALO could induce apoptosis of $\mathrm{PCa}$ in vitro and in vivo.

Most important is that our in vivo study demonstrated that ALO per o.s. daily is an effective way to inhibit PCa xenograft growth without serious side effects for the mice. In other words, ALO may be a promising therapeutic agent for $\mathrm{PCa}$.

\section{Conclusion}

Our study demonstrated that ALO executed antitumor effects against $\mathrm{PCa}$ through the induction of apoptosis and cell cycle arrest both in vitro and in vivo. The potential molecular mechanisms by which ALO suppressed the PCa growth may involve the inhibition of the phosphorylation of Akt and ERK and the activation of P53/P21 pathway and cleaved caspase 3 . We believe that ALO may be a promising candidate for the development of antitumor drugs targeting PCa.

\section{Ethics approval}

The study was approved by the Animal Care Committee of the medical school of Southeast University, and mice were treated according to the National Institutes of Health Guide for the Care and Use of Laboratory Animals.

\section{Acknowledgments}

This work was supported by grants from National Natural Science Foundation of China (No 81672551, 81572517, 81370849 and 81300472), Natural Science Foundation of Jiangsu Province (BK20161434, BL2013032, BK20150642 and BK2012336), Six Talent Peaks Project in Jiangsu Province, Jiangsu Provincial Medical Innovation Team (CXTDA2017025), Jiangsu Provincial Medical Talent (ZDRCA2016080), Jiangsu Provincial Medical Youth Talent 
(QNRC2016821, QRNC2016820) and Graduate Research Innovation Program (KYCX17_0180).

\section{Disclosure}

The authors report no conflicts of interest in this work.

\section{References}

1. Siegel RL, Miller KD, Jemal A. Cancer statistics, 2018. CA Cancer J Clin. 2018;68(1):7-30.

2. Chen W, Zheng R, Baade PD, et al. Cancer statistics in China, 2015. CA Cancer J Clin. 2016;66(2):115-132.

3. Korpal M, Korn JM, Gao X, et al. An F876L mutation in androgen receptor confers genetic and phenotypic resistance to MDV3100 (enzalutamide). Cancer Discov. 2013;3(9):1030-1043.

4. Nelson WG, Yegnasubramanian S. Resistance emerges to secondgeneration antiandrogens in prostate cancer. Cancer Discov. 2013;3(9): 971-974.

5. Zhou CC, Gao HB, Sun XB, et al. [Anti-inflammatory and anti-allergic action of aloperine]. Zhongguo Yao Li Xue Bao. 1989;10(4):360-365 Chinese [with English abstract].

6. Yuan XY, Liu W, Zhang P, Wang RY, Guo JY. Effects and mechanisms of aloperine on 2, 4-dinitrofluorobenzene-induced allergic contact dermatitis in BALB/c mice. Eur J Pharmacol. 2010;629(1-3):147-152.

7. Ma NT, Zhou R, Chang RY, et al. Protective effects of aloperine on neonatal rat primary cultured hippocampal neurons injured by oxygen-glucose deprivation and reperfusion. $J$ Nat Med. 2015;69(4): 575-583.

8. Hu S, Zhang $\mathrm{Y}$, Zhang $\mathrm{M}$, et al. Aloperine protects mice against ischemia reperfusion (IR)-induced renal injury by regulating PI3K/AKT/mTOR signaling and AP-1 activity. Mol Med. Epub 2015 Nov 3.

9. Zhang L, Zheng Y, Deng H, Liang L, Peng J. Aloperine induces G2/M phase cell cycle arrest and apoptosis in HCT116 human colon cancer cells. Int J Mol Med. 2014;33(6):1613-1620.

10. Wang H, Yang S, Zhou H, et al. Aloperine executes antitumor effects against multiple myeloma through dual apoptotic mechanisms. J Hematol Oncol. 2015;8:26.

11. Xu Z, Yan Y, Zeng S, et al. Reducing autophagy and inducing G1 phase arrest by aloperine enhances radio-sensitivity in lung cancer cells. Oncol Rep. Epub 2017 Jun 19.

12. Chen S, Jin Z, Dai L, et al. Aloperine induces apoptosis and inhibits invasion in MG-63 and U2OS human osteosarcoma cells. Biomed Pharmacother. 2018;97:45-52.

13. Ling $Z$, Wang $X$, Tao $T$, et al. Involvement of aberrantly activated HOTAIR/EZH2/miR-193a feedback loop in progression of prostate cancer. J Exp Clin Cancer Res. 2017;36(1):159.

14. Strozyk E, Kulms D. The role of AKT/mTOR pathway in stress response to UV-irradiation: implication in skin carcinogenesis by regulation of apoptosis, autophagy and senescence. Int J Mol Sci. 2013; 14(8):15260-15285.
15. Cagnol S, Chambard JC. ERK and cell death: mechanisms of ERKinduced cell death - apoptosis, autophagy and senescence. FEBS J. 2010;277(1):2-21.

16. McGowan EM, Tran N, Alling N, Yagoub D, Sedger LM, MartinielloWilks R. p14ARF post-transcriptional regulation of nuclear cyclin D1 in MCF-7 breast cancer cells: discrimination between a good and bad prognosis? PLoS One. 2012;7(7):e42246.

17. Shiragami R, Murata S, Kosugi C, et al. Enhanced antitumor activity of cerulenin combined with oxaliplatin in human colon cancer cells. Int J Oncol. 2013;43(2):431-438.

18. Ho JS, Ma W, Mao DY, Benchimol S. p53-Dependent transcriptional repression of c-myc is required for G1 cell cycle arrest. Mol Cell Biol. 2005;25(17):7423-7431.

19. Song XL, Zhang YJ, Wang XF, et al. Casticin induces apoptosis and G0/G1 cell cycle arrest in gallbladder cancer cells. Cancer Cell Int. 2017;17:9.

20. Kim MS, Kim JE, Lim DY, et al. Naproxen induces cell-cycle arrest and apoptosis in human urinary bladder cancer cell lines and chemically induced cancers by targeting PI3K. Cancer Prev Res (Phila). 2014;7(2): 236-245.

21. Huang WS, Kuo YH, Kuo HC, et al. CIL-102-Induced Cell Cycle Arrest and Apoptosis in Colorectal Cancer Cells via Upregulation of p21 and GADD45. PLoS One. 2017;12(1):e0168989.

22. Williams GH, Stoeber K. The cell cycle and cancer. J Pathol. 2012; 226(2):352-364.

23. Planas-Silva MD, Weinberg RA. The restriction point and control of cell proliferation. Curr Opin Cell Biol. 1997;9(6):768-772.

24. Lloberas J, Celada A. p21(waf1/CIP1), a CDK inhibitor and a negative feedback system that controls macrophage activation. Eur J Immunol. 2009;39(3):691-694.

25. Harper JW, Elledge SJ, Keyomarsi K, et al. Inhibition of cyclindependent kinases by p21. Mol Biol Cell. 1995;6(4):387-400.

26. Agarwal ML, Agarwal A, Taylor WR, Stark GR. p53 controls both the G2/M and the G1 cell cycle checkpoints and mediates reversible growth arrest in human fibroblasts. Proc Natl Acad Sci U S A. 1995;92(18): 8493-8497.

27. Giono LE, Manfredi JJ. The p53 tumor suppressor participates in multiple cell cycle checkpoints. J Cell Physiol. 2006;209(1):13-20.

28. Bologna-Molina R, Mosqueda-Taylor A, Molina-Frechero N, MoriEstevez AD, Sanchez-Acuna G. Comparison of the value of PCNA and Ki-67 as markers of cell proliferation in ameloblastic tumors. Med Oral Patol Oral Cir Bucal. 2013;18(2):e174-e179.

29. $\mathrm{Hu} \mathrm{H}$, Jiang $\mathrm{C}, \mathrm{Li} \mathrm{G}, \mathrm{Lu}$ J. PKB/AKT and ERK regulation of caspasemediated apoptosis by methylseleninic acid in $\mathrm{LNCaP}$ prostate cancer cells. Carcinogenesis. 2005;26(8):1374-1381.

30. Yang B, Johnson TS, Thomas GL, et al. A shift in the Bax/Bcl-2 balance may activate caspase-3 and modulate apoptosis in experimental glomerulonephritis. Kidney Int. 2002;62(4):1301-1313.
OncoTargets and Therapy

\section{Publish your work in this journal}

OncoTargets and Therapy is an international, peer-reviewed, open access journal focusing on the pathological basis of all cancers, potential targets for therapy and treatment protocols employed to improve the management of cancer patients. The journal also focuses on the impact of management programs and new therapeutic agents and protocols on

\section{Dovepress}

patient perspectives such as quality of life, adherence and satisfaction. The manuscript management system is completely online and includes a very quick and fair peer-review system, which is all easy to use. Visit http://www.dovepress.com/testimonials.php to read real quotes from published authors. 